\title{
Long-period Gratings in Chemical Sensing
}

\author{
Jack A. Barnes, R. Stephen Brown, Judy Cipot-Wechsler, Cathleen M. Crudden, Jenny Du, Hans- \\ Peter Loock*, Krista Plett \\ Department of Chemistry, Queen's University Kingston, ON, K7L 3N6, Canada
}

\begin{abstract}
A chemical sensor system consisting of a coated long period grating, which was spliced into a fiber loop cavity, has been prepared and characterized. Designer coatings based on polydimethylsiloxane and nanostructured organically modified silica (ORMOSIL) materials were prepared to provide enhanced sensitivity for a variety of key environmental pollutants. Upon microextraction of the analyte into the polymer matrix, an increase in the refractive index of the coating resulted in a change of the attenuation spectrum of the long period grating. The grating was interrogated using ring-down detection as a means to amplify the optical loss and to gain stability against misalignment and laser power fluctuations. Chemical differentiation of cyclohexane and xylenes was achieved and a detection limit of $300 \mathrm{ppm}$ of xylenes vapour in air was readily realized for PDMS coatings. Ormosil-type coatings were capable of detecting lead cations at concentrations below 1 ppm in water.
\end{abstract}

Keywords: Long-period grating (LPG), solid-phase microextraction (SPME), chemical sensor, cavity ring-down (CRD), fiber-loop ring-down (FLRD), mesoporous silica (SBA-15), mesoporous silicate.

\section{INTRODUCTION}

In recent years, many fiber-optic sensors have been developed for field applications in temperature, pressure and humidity sensing. ${ }^{1,2}$ (Bio-)chemical sensors have also been developed and display impressive detection limits and sensitivity in lab environments. Many waveguide based-sensors measure the optical loss that is directly or indirectly correlated to the analyte concentration of interest whereas others are based on fluorescence detection. Refractive index sensors are a third class of chemical sensors and are likely to be the most general, since refractive index is a universal optical property that is exhibited by all materials and may be probed at any wavelength - including the telecom bands. Long-period gratings (LPGs) have been shown to be sensitive refractive index sensors, ${ }^{3,4,5,6,7}$ but by themselves LPGs do not provide the sensitivity and chemical specificity that is needed for an effective chemical sensor. As shown in this report, coating the LPGs with functionalized materials can increase the concentration of the desired analyte in the immediate vicinity of the grating surface by 2 to 6 orders of magnitude, and can provide some chemical specificity. Since the coating's refractive index changes with uptake of the analyte, the transmission spectrum of the grating shifts and may be monitored either by using a broadband light source in combination with a spectrum analyzer or - as demonstrated below - by a narrow-band light source in combination with a broad band detector. In our sensor system, we amplify the optical loss induced by the grating by placing the LPG into an optical cavity and measuring the lifetime of photons in the cavity. Such a cavity ring-down time measurement not only provides for increased sensitivity but also for an optical loss measurement that is independent of light source power fluctuations or detector response drifts and is consequently especially useful for field measurements. ${ }^{8}$

With this report we present a proof-of-concept sensor system that has been developed with the ultimate aim of deployment at groundwater monitoring wells in mind. While the demands on sensitivity, detection limit and chemical specificity are somewhat relaxed for this application, the sensor has to be rugged, compact and inexpensive.

\section{EXPERIMENT}

The chemical sensor consists of three components - the Long-Period Grating (LPG), a solid-phase microextraction (SPME) coating, and fiber-loop ring-down (FLRD) detection - that are each optimized to provide for superior sensitivity, while preserving the system's ruggedness and field suitability. They will be discussed separately in the following

Photonics North 2008, Réal Vallée, Michel Piché, Peter Mascher, Pavel Cheben, Daniel Côté,

Sophie LaRochelle, Henry P. Schriemer, Jacques Albert, Tsuneyuki Ozaki, Eds., Proc. of SPIE

Vol. 7099, 70992C, (2008) - 0277-786X/08/\$18 · doi: 10.1117/12.806722

Proc. of SPIE Vol. 7099 70992C-1 
paragraphs. The performance of the sensor system will be illustrated with two examples, i.e. gas-phase sensing of volatile organic compounds (VOCs) using polymer coatings, and detection of lead in aqueous solution.

\subsection{Long-period grating}

A long period grating is a modulation of refractive index with period $\Lambda$ written into the core of a single-mode waveguide. When the wavelength, $\lambda$, of the light guided by the fiber fulfills the phase-matching condition,

$$
\lambda_{i}=\Lambda\left[n_{\text {eff } f_{\text {core }}}\left(\lambda, n_{1}, n_{2}\right)-n_{\text {eff clading }}^{i}\left(\lambda, n_{1}, n_{2}, n_{3}\right)\right]
$$

light is efficiently coupled into the cladding of the waveguide and is attenuated. Here $n_{\text {eff } \text { fore }}$ is the refractive index of the propagated core mode whereas $n_{\text {eff chadinin }}^{i}$ is the effective refractive index of cladding mode $i$. LPGs have found uses as band rejection filters and as gain-flatting filters for fiber amplifiers. As is apparent from the above equation, the rejection wavelength depends on the effective refractive index of the cladding modes, $n_{\text {eff claditing }}^{i}$, which is a function of the refractive index of the immediate environment of the LPG, $n_{3}$. Here, the higher order cladding modes, which have a larger evanescent field, are most strongly affected. The sensitivity of an LPG then depends on the period of the grating, i.e. a short period provides access to higher order cladding modes that show a stronger response to refractive index changes. In the course of this work, LPGs provided by ITF Labs, Montreal, QC, with periods of $320 \mu \mathrm{m}$ and $236 \mu \mathrm{m}$ have been employed.

It is well-known that LPGs may be used as refractive index sensors, and numerous applications for chemical sensing have been reported previously. ${ }^{9}$ As was emphasized above, the response of the LPG spectrum to a refractive index change is strongly non-linear and is maximized for ambient refractive indices that are close to but slightly below that of the cladding material. Below we illustrate how designer coatings further enhance the response to chemical contaminants.

\subsection{Coatings}

Two classes of coatings were investigated. Firstly, polymer coatings based on polydimethylsiloxane (PDMS) are largely impenetrable to water but readily absorb hydrophobic compounds such as chlorinated solvents, gasoline, and volatile organic compounds (VOCs) including other compounds of environmental concern. ${ }^{10}$ Solid-phase microextraction using PDMS has found applications mostly in sample acquisition and pre-concentration. ${ }^{11}$ Commercial PDMS has a refractive index of only $\mathrm{n}_{3}=1.39$. In order to increase the refractive index of the polymer, we synthesized polysiloxanes from different $\mathrm{SiR}_{2}(\mathrm{Cl})_{2}$ monomers, where $\mathrm{R}=$ methyl, phenyl, and octyl. Titanium(IV) tetraisopropoxide was used as a cross-linking agent and also used to increase the refractive index to about $n_{3}=1.44$. Adjustment of the refractive index to this range resulted in a dramatic increase in the sensitivity of the LPG sensor.

A second class of coatings was prepared from nanostructured organically modified silica materials. Mesoporous silicas can be synthesized with a highly ordered, open-pore structure that is readily penetrated by water. Functionalization of these mesoporous silicas with e.g. thiol, amino, or phosphoester groups has led to materials that are capable of extracting large amounts of heavy metals from aqueous solution while remaining insensitive to common interferences such as sodium chloride. ${ }^{12}$

Materials such as those investigated in the current study have demonstrated an uptake of $30 \%$ by weight in mercury $\left(\mathrm{Hg}^{2+}\right) .{ }^{13,14,15}$ Here, the coatings were prepared by co-condensation of $\mathrm{Si}(\mathrm{OEt})_{4}$ and $(\mathrm{EtO})_{3} \mathrm{Si}-\mathrm{R}-\mathrm{Si}(\mathrm{OEt})_{3}$ precursors in the presence of a surfactant. Using the surfactant templating approach ${ }^{16,17,18}$, the hydrophilic silica precursors interact with the hydrophilic heads groups of the surfactants to adopt a liquid crystalline mesophase via cooperative selfassembly. After condensation of the precursors to form the silica matrix, the template can be removed to yield a porous, nanostructured material. The details of the material synthesis will be described in a separate communication. 


\subsection{Fiber-loop ring-down detection}

Fiber loop ring-down measurements of optical loss are a simple and yet powerful method of amplifying and determining optical loss in a fiber optic waveguide. ${ }^{19,20}$ The method is derived from cavity ring-down spectroscopy, an established gas-phase absorption spectroscopic method. ${ }^{21}$ In fiber loop ring-down spectroscopy, a light pulse is injected into a loop of optical waveguide containing a sensing element such as the long-period grating described above. The light pulse will traverse the sensor element many times before its intensity decays below the detection threshold. The exponential decay of the intensity in the loop is monitored and the optical loss is inferred from the exponential decay time,

$$
\tau=\frac{4.34 t_{R}}{A(d B)}
$$

where $t_{R}=n L / c_{0}$ is the roundtrip time of a pulse in a loop of length $L$ and with refractive index $n$. The ring-down method not only amplifies the measurement of the optical loss $A$, but also renders the system robust against laser power fluctuations or detector response drift, since only the rate of intensity change is measured. To further simplify the system and reduce cost, instead of using switched laser diodes we used a sine-wave amplitude modulated laser light source. ${ }^{22}$ The ring-down time can be calculated from the phase-shift,

$$
\phi=\arctan (-\Omega \tau)
$$

of the modulated light emitted from the cavity, measured with respect of the light entering the cavity. As was pointed out before $^{23}$, optimum sensitivity is obtained when the modulation frequency $\Omega=2 \pi \mathrm{f}$ is about equal to $1 / \tau$.

\subsection{System Integration}

The coated LPG is spliced into a $10 \mathrm{~m}$ loop of single mode fiber and light is coupled into the loop using a $99: 1,2 \times 2$ fiber-fiber coupler (Lightwave Technologies). The intensity in the loop is measured with a parasitic inline detector (Eigenlight). The detector output is amplified $(500 \mathrm{~V} / \mathrm{mA})$ and then directed to a phase comparator chip (Analog Devices, AD8302) (Figures 1 and 2).

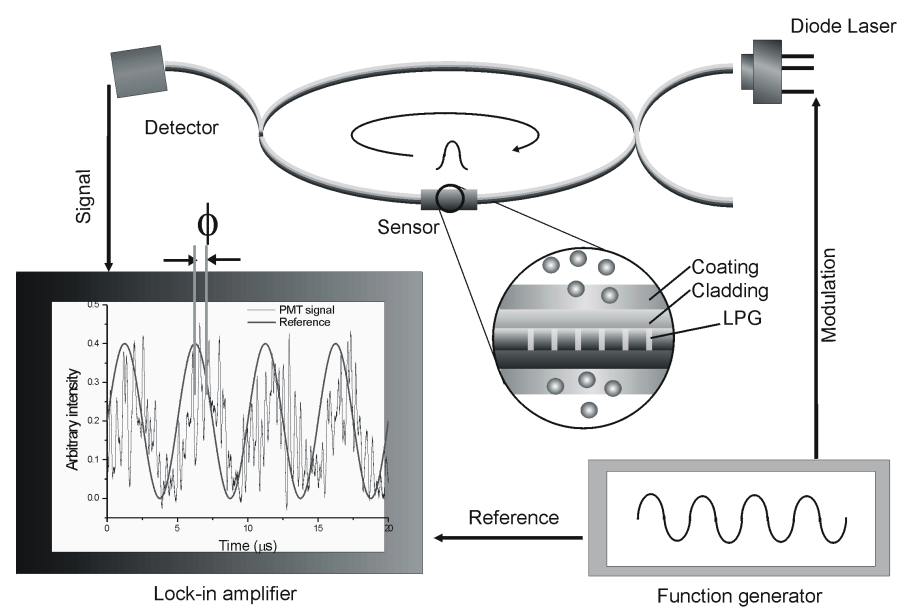

Figure 1. Experimental setup showing the single-mode fiber loop into which light from a tunable diode laser is coupled. The coated LPG is the sensing element and exposed to either a gas flow or contaminated water. The attenuation in the fiber optic loop causes a phase shift at the detector which is sampled by a phase comparator circuit. 


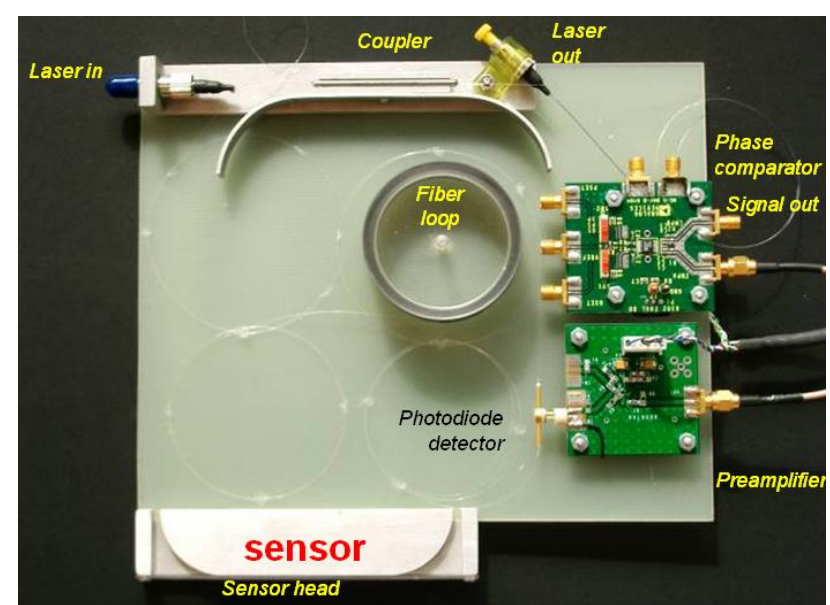

Figure 2. Photograph of the experimental setup. The light in the loop is detected using an inline parasitic InGaAs detector, amplified and then sent to a phase comparator integrated circuit.

\section{RESULTS AND DISCUSSION}

The device shown in Figure 2 has been used to determine the optical loss caused by a long-period grating as a function of external refractive index. Different DMSO/water solutions have been prepared to tune the refractive index between $\mathrm{n}_{3}=$ 1.315 and 1.466. The refractive indices of these solutions were calculated using the Lorentz-Lorenz equation ${ }^{24}$ together with previously reported refractive index values for DMSO and water of $n_{3}=1.4666^{25}$ and $n_{3}=1.31499{ }^{26}$, respectively, at $1.55 \mu \mathrm{m}$ and $25^{\circ} \mathrm{C}$. As is apparent from Figure 3(b) the LPG attenuation peak is shifted to coincide with the laser wavelength as the refractive index is increased to about 1.44. A further increase of the ambient refractive index makes the cladding modes lossy and the attenuation of the LPG is decreased at the laser wavelength. The ring-down time (Figure 3a) is calculated from the measured phase shift using equation (3). The spectra of LPGs that were coated with polysiloxanes were also recorded using a broadband lightsource and a spectrum analyzer. As is shown in Figure 4 the polymer with high phenyl content has an increased sensitivity to compounds such as xylenes and cyclohexane. This is primarily due to the increase of the refractive index of the coating into the range of greater sensitivity for the LPG. Furthermore, it is apparent that cyclohexane, with a refractive index which is lower than that of the high phenyl content coating, decreases the coating refractive index with increasing uptake. Both effects may be used to distinguish and quantify cyclohexane and xylenes.
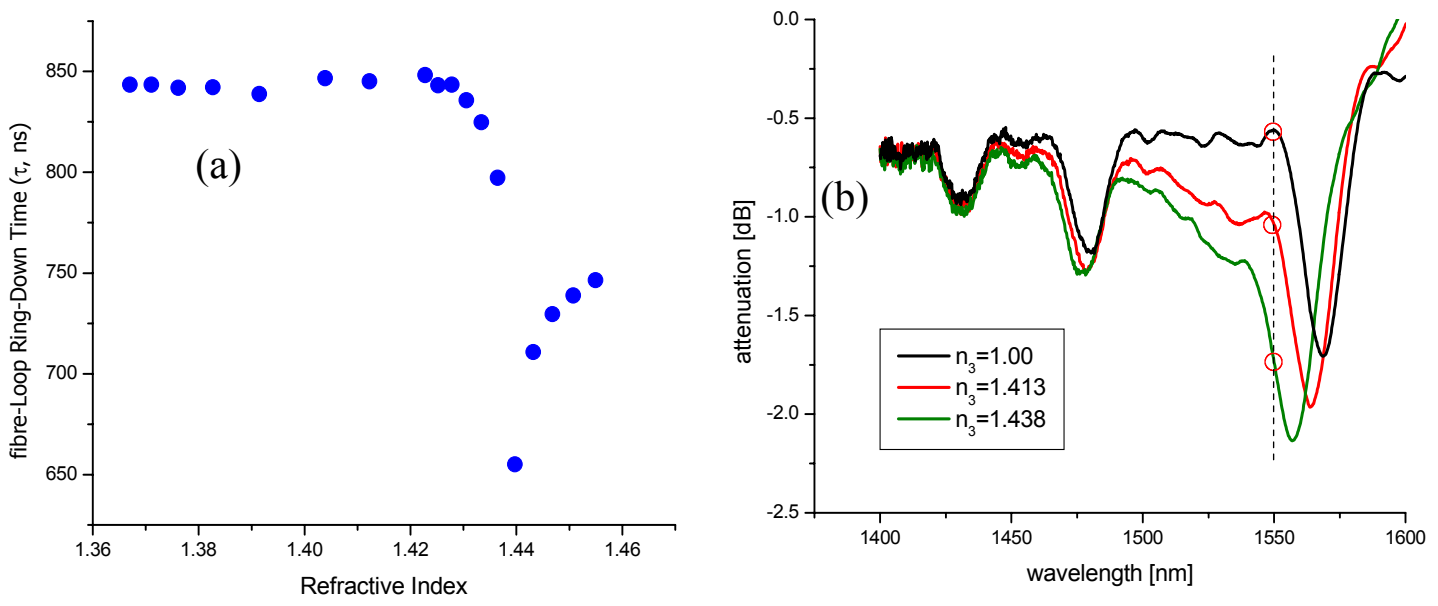

Figure 3. (a) Response of the device shown in Figure 2 to a changing ambient refractive index. (b) As the LPG's attenuation peak shifts to shorter wavelength the attenuation of the $1550 \mathrm{~nm}$ laser light is increased and the ringdown-time is decreased. 

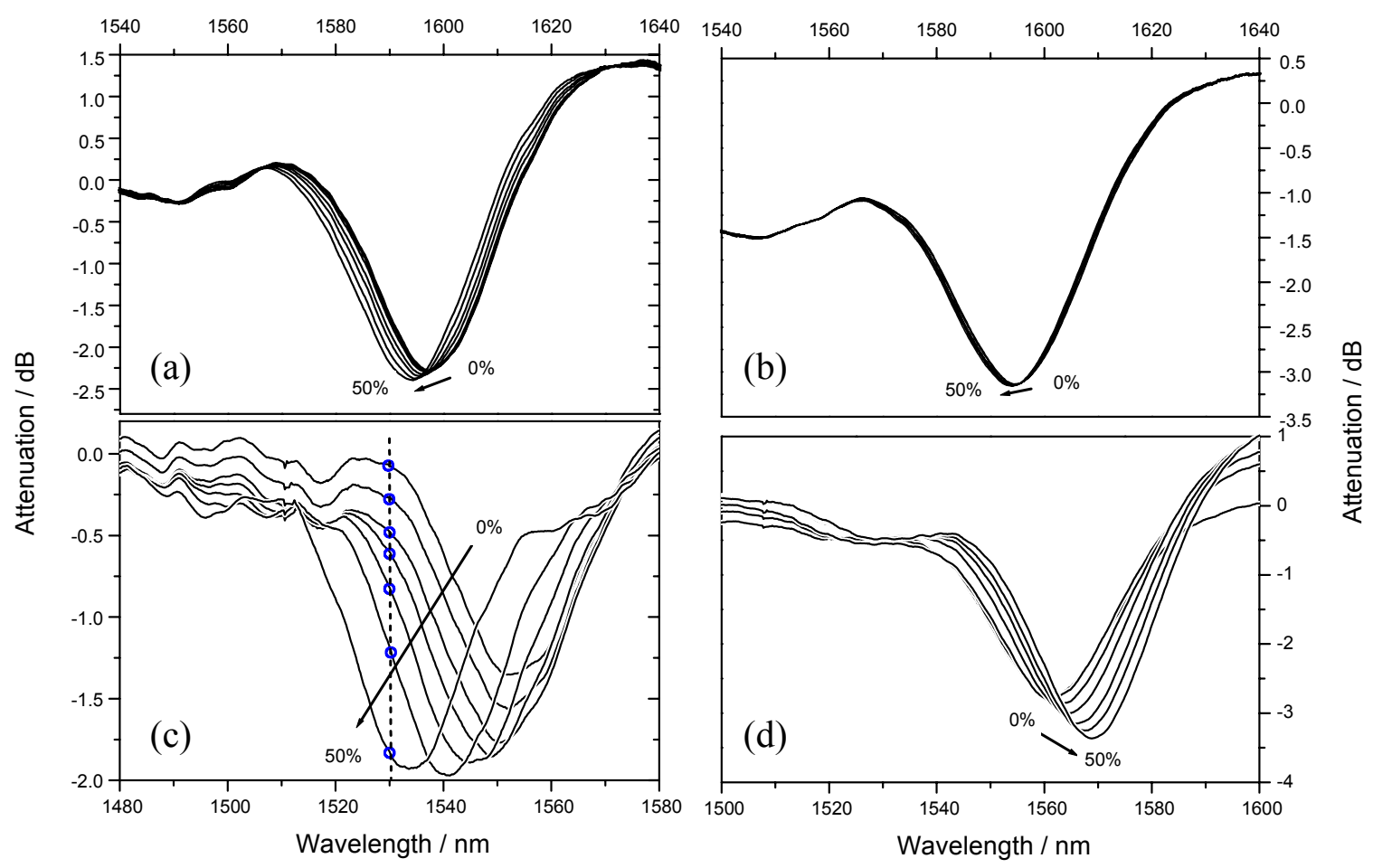

Figure 4. (a) Response of the device shown in Figure 2 to xylenes vapour (a and c) and to cyclohexane vapour (b and d). The concentrations are given with respect to the respective saturation partial pressures of these VOCs in air at 20 C. Panels (a) and (b) show the spectrum of a $320 \mu \mathrm{m}$ LPG that is coated with commercial PDMS (Sylgard 184) of refractive index $n_{3}=1.39$. Panels (c) and (d) illustrate the enhanced response when the PDMS polymer is modified with $8.5 \%$ diphenyl siloxane. The circles in (c) highlight the concentration dependent LPG attenuation losses at the interrogation wavelength of $1530 \mathrm{~nm}$.
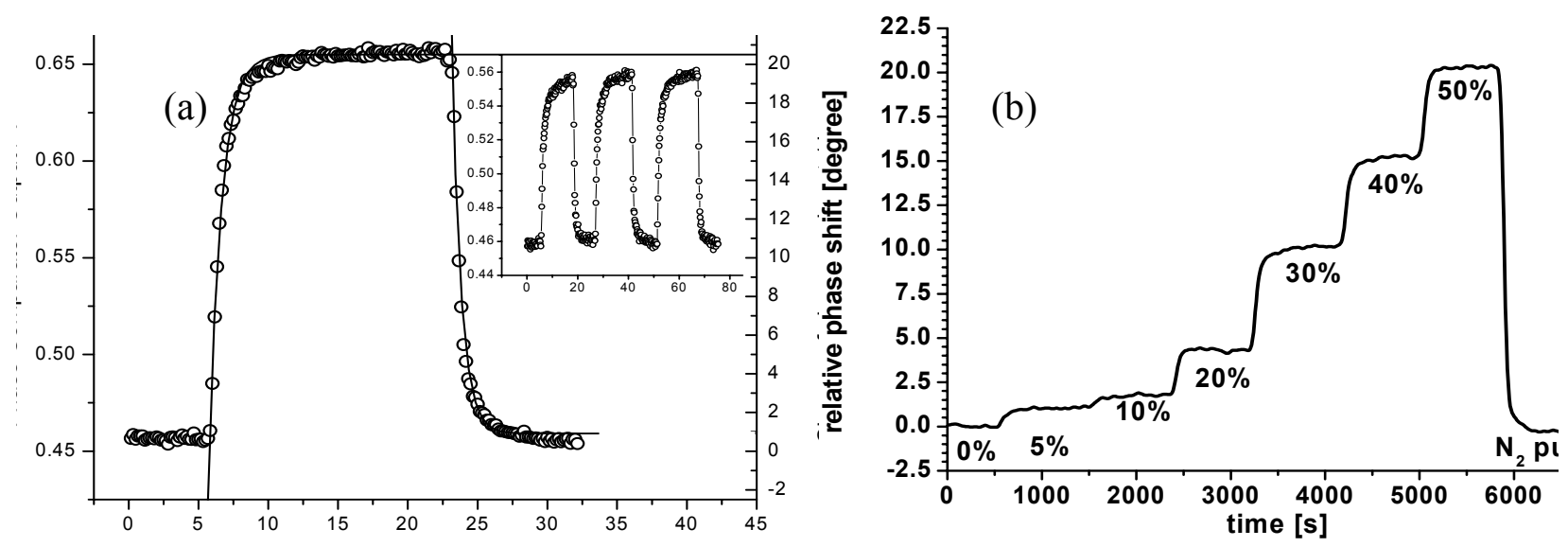

Figure 5. (a) Response of the PDMS coated LPG substituted with 8.5\% diphenyl siloxane, which is integrated in the system shown in Figure 2, to a sudden increase of xylenes vapour pressure. The pressure of xylenes in air was increased to $50 \%$ of its saturation pressure $(4.2$ Torr or $5500 \mathrm{ppm})$ and then reduced to zero. Three such exposure cycles are shown in the insert. (b) The LPG's attenuation at $1530 \mathrm{~nm}$ to various xylenes levels is strongly nonlinear with increasing xylenes concentration as expected from Figure 4c. 
When incorporated into the fiber loop and interrogated at about $1530 \mathrm{~nm}$, there is a dramatic change in the modulation phase shift as the xylenes are partitioned into the polymer coating. The uptake is reversible and has a time constant of less than one minute. The inset in Fig 5(a) illustrates that the process is reproducible.

Mesoporous silica films were prepared by co-condensation of $\mathrm{Si}(\mathrm{EtO})_{4}$ with $\mathrm{RSi}(\mathrm{EtO})_{4}$ in the presence of a structuredirecting agent (Brij 56). A transmission electron micrograph (Figure 6a) shows that a hexagonal pore pattern is created in which the cylindrical pores have a diameter of about $10 \mathrm{~nm}$. Using a triblock copolymer (Pluronic ${ }^{\circledR}$ F127) instead of the alkylpolyether, a uniform, interconnected wormhole structure is obtained after dip-coating a film onto a single mode fibre, and the porosity is retained after solvent extraction with acetone to remove the template (Fig. 6b). The thickness of the film is about $2 \mu \mathrm{m}$ as estimated from the scanning electron micrograph (Fig. 6c). Preliminary measurements indicate a high partitioning coefficient of lead $\mathrm{Pb}^{2+}$ into the thiol-functionalized film $(\mathrm{pK}>5)$, which was found to lead to a strong response of an LPG that was coated with the film (Figure 7). A detection limit of under $1 \mathrm{ppm}^{2+} \mathrm{b}^{2+}$ in aqueous solution was realized. Incorporation of the mesoporous silica coated LPG into the fiber loop is under way.

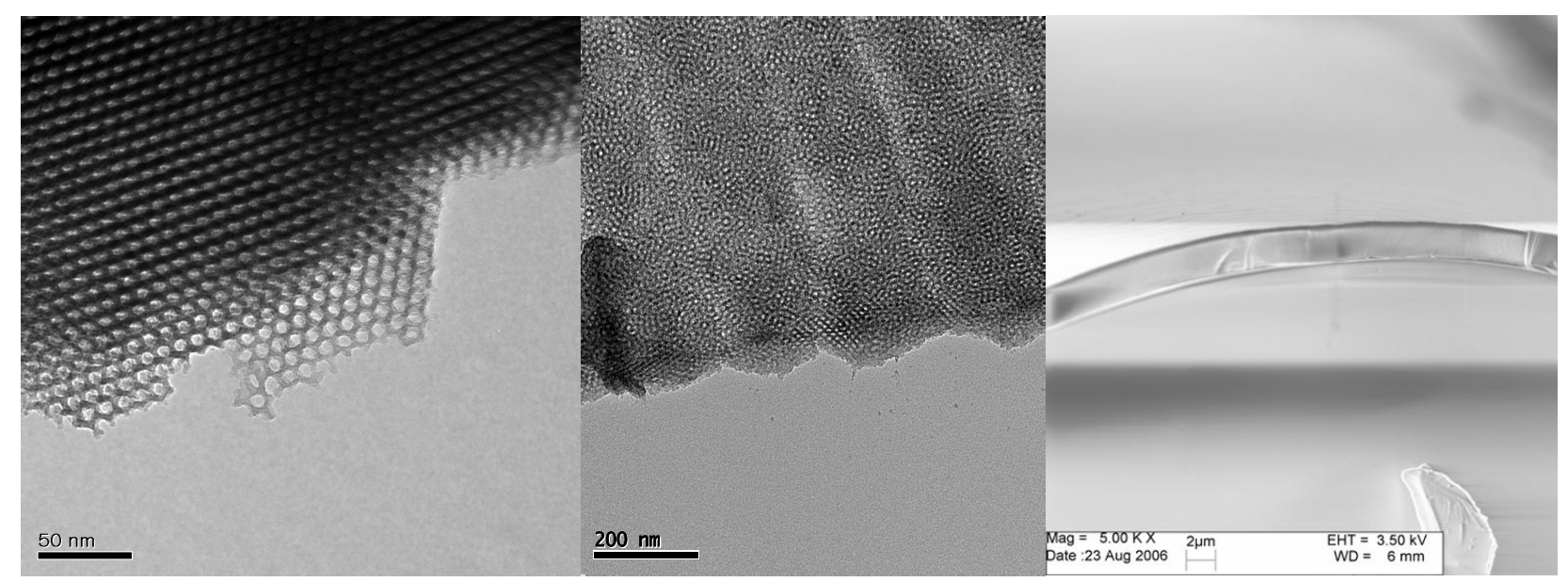

Figure 6. (a) Scanning electron micrograph (SEM) of a mesoporous silica film that was grown on a planar glass substrate (b) A similar film grown on a SMF-28 waveguide (c) SEM of a film on the SMF fiber at lower magnification.

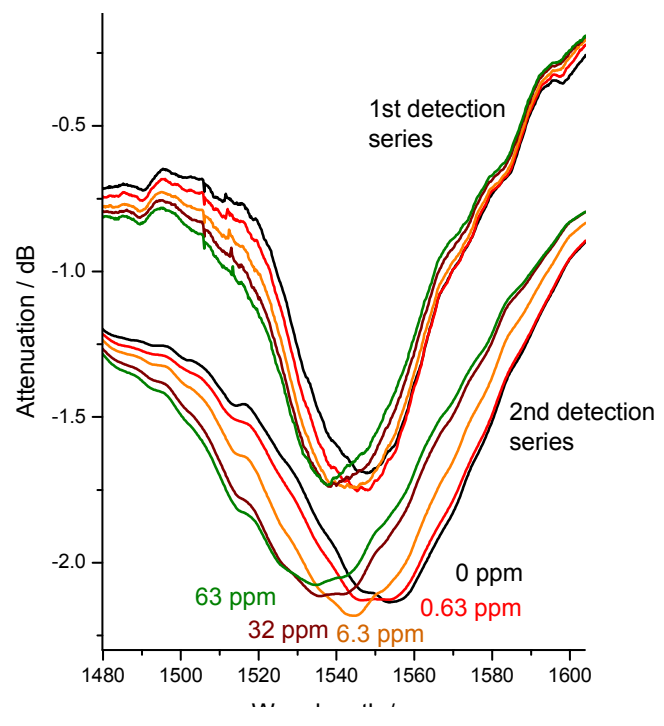

Figure 7. Attenuation spectrum of an LPG that was coated with the film shown in Figure 6. To adjust the refractive index of the film, $\mathrm{Pb}^{2+}$ was detected in a $40 \%$ solution of DMSO in water. The LPG spectrum shows a marked shift even at concentrations below $1 \mathrm{ppm}$. 


\section{CONCLUSIONS}

The preliminary results in this report not only demonstrate the feasibility of a rugged and stand-alone fiber-optic chemical sensor but also point out avenues for further improvements. For example, a lower detection limit and higher sensitivity are possible by using different classes of coating materials that are better matched to the refractive index of the waveguide material and that have higher partitioning coefficients for the desired analyte targets. Higher order cladding modes show greater sensitivity towards refractive index changes and may be accessed by using a reduced grating period and/or reduced fiber diameter. Finally, our preliminary work on the detection of heavy metal ions with mesoporous silica coatings needs to be expanded to include other ions. While both classes of coatings are expected to be insensitive to common interferences $\left(\mathrm{Na}^{+}, \mathrm{Ca}^{2+}, \mathrm{K}^{+}, \mathrm{Cl}^{-}\right)$, only rigorous tests will confirm whether the sensor systems is indeed ready for field deployment.

\section{REFERENCES}

1 Lee, B., "Review of the present status of optical fiber sensors", Opt. Fiber Technol. 9, 57 (2003)

2 Kersey, A. D., Davis, M. A., Patrick, H. J., LeBlanc, M., Koo, K. P., Askins, C. G., Putnam, M. A., and Friebele, E. J., "Fiber grating sensors", J Lightwave Technol. 15, 1442 (1997)

3 Patrick, H. J., Kersey, A. D., and Bucholtz, F., "Analysis of the response of long period fiber gratings to external index of refraction", J Lightwave Technol. 16, 1606 (1998)

4 Bhatia, V., "Applications of long-period gratings to single and multi-parameter sensing", Opt. Express 4, 457 (1999)

5 Chong, J. H., Shum, P., Haryono, H., Yohana, A., Rao, M. K., Lu, C., and Zhu, Y. N., "Measurements of refractive index sensitivity using long-period grating refractometer", Opt. Commun. 229, 65 (2004)

6 Lee, S. T., Kumar, R. D., Kumar, P. S., Radhakrishnan, P., Vallabhan, C. P. G., and Nampoori, V. P. N., "Long period gratings in multimode optical fibers: application in chemical sensing", Opt. Commun. 224, 237 (2003)

7 Shu, X. W. and Huang, D. X., "Highly sensitive chemical sensor based on the measurement of the separation of dual resonant peaks in a 100-mu m-period fiber grating", Opt. Commun. 171, 65 (1999)

8 Tong, Z. G., Wright, A., McCormick, T., Li, R. K., Oleschuk, R. D., and Loock, H.-P., "Phase-shift fiber-loop ringdown spectroscopy", Anal. Chem. 76, 6594 (2004)

9 James, S. W. and Tatam, R. P., "Optical fibre long-period grating sensors: Characteristics and application", Meas. Sci. Technol. 14, R49 (2003)

10 Eisert, R. and Levsen, K., "Solid-phase microextraction coupled to gas chromatography: A new method for the analysis of organics in water", J. Chromatogr. A 733, 143 (1996)

11 Pawliszyn, J., "Solid Phase Microextraction", (1997) Wiley-VCH

12 Feng, X., Fryxell, G. E., Wang, L. Q., Kim, A. Y., Liu, J., and Kemner, K. M., "Functionalized monolayers on ordered mesoporous supports", Science 276, 923 (1997)

13 Mercier, L. and Pinnavaia, T. J., "Access in mesoporous materials: Advantages of a uniform pore structure in the design of a heavy metal ion adsorbent for environmental remediation", Adv. Mater. 9, 500 (1997)

14 Zhang, L. X., Zhang, W. H., Shi, J. L., Hua, Z., Li, Y. S., and Yan, J., "A new thioether functionalized organicinorganic mesoporous composite as a highly selective and capacious $\mathrm{Hg}^{2+}$ adsorbent", Chem. Comm. 210 (2003)

15 Fryxell, G. E., "The synthesis of functional mesoporous materials", Inorg. Chem. Comm. 9, 1141 (2006)

16 Yanagisawa, T., Shimizu, T., Kuroda, K., and Kato, C., "The Preparation of Alkyltrimethylammonium-Kanemite Complexes and Their Conversion to Microporous Materials", Bull. Chem .Soc. Japan 63, 988 (1990)

17 Kresge, C. T., Leonowicz, M. E., Roth, W. J., Vartuli, J. C., and Beck, J. S., "Ordered Mesoporous MolecularSieves Synthesized By A Liquid-Crystal Template Mechanism", Nature 359, 710 (1992)

18 Zhao, D. Y., Feng, J. L., Huo, Q. S., Melosh, N., Fredrickson, G. H., Chmelka, B. F., and Stucky, G. D., "Triblock copolymer syntheses of mesoporous silica with periodic 50 to 300 angstrom pores", Science 279, 548 (1998)

19 Brown, R. S., Kozin, I., Tong, Z., Oleschuk, R. D., and Loock, H.-P., "Fiber-loop ring-down spectroscopy", J. Chem. Phys. 117, 10444 (2002)

20 Tong, Z, Jakubinek, M, Wright, A, Gillies, A, and Loock, H.-P., "Fiber-loop ring-down spectroscopy: A sensitive absorption technique for small liquid samples", Rev. Sci. Instrum. 74, 4818 (2003)

21 Mazurenka, M., Orr-Ewing, A. J., Peverall, R., and Ritchie, G. A. D., "Cavity ring-down and cavity enhanced spectroscopy using diode lasers", Annu. Rep. Prog. Chem., Sect. C 101, 100 (2005)

22 Tong, Z, Wright, A, McCormick, T, and Loock, H.-P., "Phase shift fiber-loop ring-down spectroscopy", Anal. Chem. 76, 6594 (2004) 
23 Engeln, R., von Helden, G., Berden, G., and Meijer, G., "Phase shift cavity ring down absorption spectroscopy", Chem. Phys. Lett. 262, 105 (1996)

24 Mehra, R., "Application of refractive index mixing rules in binary systems of hexadecane and heptadecane with nalkanols at different temperatures", Proc .Indian Acad. Sciences - Chem. Sciences 115, 147 (2003)

25 Kozma, I. Z., Krok, P., and Riedle, E., "Direct measurement of the group-velocity mismatch and derivation of the refractive-index dispersion for a variety of solvents in the ultraviolet", J. Opt .Soc. Am. B - Opt. Phys. 22, 1479 (2005)

26 Harvey, A. H., Gallagher, J. S., and Sengers, J. M. H. L., "Revised formulation for the refractive index of water and steam as a function of wavelength, temperature and density", J. Phys. Chem. Ref. Data 27, 761 (1998) 